\title{
FLAIR vascular hyperintensity: an unfavorable marker of early neurological deterioration and short-term prognosis in acute ischemic stroke patients
}

\author{
Li Zhu ${ }^{1,2}$, Shenchu Gong ${ }^{2}$, Xiangyang Zhu ${ }^{3}$, Ru Zhang ${ }^{2}$, Kaixuan Ren ${ }^{2}$, Zhengqi Zhu' ${ }^{2}$ Tianle Wang ${ }^{2}$, \\ Wei Xing'
}

${ }^{1}$ Department of Radiology, The Third Affiliated Hospital of Soochow University, Changzhou, China; ${ }^{2}$ Department of Radiology, ${ }^{3}$ Department of Neurology, The Second Affiliated Hospital of Nantong University, Nantong, China

Contributions: (I) Conception and design: L Zhu; (II) Administrative support: W Xing; (III) Provision of study materials or patients: X Zhu, S Gong; (IV) Collection and assembly of data: R Zhang, K Ren, Z Zhu; (V) Data analysis and interpretation: L Zhu, T Wang; (VI) Manuscript writing: All authors; (VII) Final approval of manuscript: All authors.

Correspondence to: Wei Xing. Department of Radiology, The Third Affiliated Hospital of Soochow University, Changzhou 213000, China.

Email: suzhxingwei@suda.edu.cn; Tianle Wang. Department of Radiology, The Second Affiliated Hospital of Nantong University, Nantong 226001,

China. Email: wangtianle9192@163.com.

Background: To investigate the value of fluid-attenuated inversion recovery (FLAIR) Vascular Hyperintensity (FVH) in predicting early neurological deterioration (END) and short-term prognosis in acute ischemic stroke (AIS) patients who beyond the time window for recanalization therapy.

Methods: We retrospectively analyzed the AIS patients from 24 to 72 hours after symptom onset, who received dual antiplatelet therapy (DAPT). The patients were divided into the END and no early neurological deterioration (NEND) group according to the change of the National Institutes of Health Stroke Scale (NIHSS) score. The patients were also divided into the favorable and unfavorable prognosis group according to the 90 day modified Rankin Scale (mRS). The Alberta Stroke Program Early CT Score (ASPECTS) was used to assess the scope of infarction on DWI; the modified ASPECTS was used to assess the presence of FVH on FLAIR and multiple hypointense vessels (MHV) on SWI. We performed binary stepwise regression analysis with END and short-term prognosis as dependent variables to evaluate the odds ratio (OR) and its $95 \%$ confidence interval (CI) of primary outcomes. Next, we sequentially excluded nonsignificant variables from the last model to determine the risk factors of END.

Results: Two-hundred sixty-seven patients were included in this study. The median NIHSS score at admission was 6 [interquartile range (IQR) 5,9], the median DWI-ASPECTS at admission was 8 (IQR 6, 9), the median FVH score was 7 (IQR 3, 7), and the median MHV-ASPECTS was 8 (IQR 6, 8). The NIHSS score at admission was higher in the END group. The MHV-ASPECTS, DWI-ASPECTS, and FVH-ASPECTS were lower in the END group. Binary stepwise regression analysis showed that the FVHASPECTS (OR =0.39, 95\% CI: 0.174-0.872) and vascular stenosis/occlusion (OR =0.015, 95\% CI: 0.0000.943 ) were independent risk factors of END.

Conclusions: For AIS patients beyond the time window for recanalization therapy who are receiving DAPT, a low FVH-ASPECTS is associated with a higher risk of END. In patients with vascular occlusion/ stenosis, FVH may be used as a predictor of END and an unfavorable 90-day prognosis in patients beyond the time window for recanalization therapy who are receiving DAPT.

Keywords: Acute ischemic stroke (AIS); early neurological deterioration (END); fluid-attenuated inversion recovery (FLAIR) vascular hyperintensity $(\mathrm{FVH})$

Submitted Apr 25, 2020. Accepted for publication Jul 08, 2020.

doi: 10.21037/apm-20-1175

View this article at: http://dx.doi.org/10.21037/apm-20-1175 


\section{Introduction}

About one-third of patients with acute ischemic stroke (AIS) experience early neurological deterioration (END) (1). Once it occur, END often leads to severe neurological dysfunction (2). The National Institutes of Health Stroke Scale (NIHSS) score at admission, symptomatic cerebral hemorrhage, infarction volume, and malignant edema are risk factors of END (3), whereas hemodynamic changes and perfusion abnormalities may be the key factors inducing END (4).

Fluid-attenuated inversion recovery (FLAIR) vascular hyperintensity $(\mathrm{FVH})$ indirectly reflects the state of cerebral blood flow of AIS patients and is a critical marker for collateral circulation after large-vessel occlusion (5). Its value in predicting the short-term prognosis of stroke patients is still a controversial topic (6-11). Studies on the predictive value of FVH have focused on patients who received reperfusion within 24 hours of stroke onset. However, $10 \%$ to $15 \%$ of AIS patients are unable to receive early and effective reperfusion in clinical practice (12). For AIS patients beyond the time window for thrombolysis who are receiving dual antiplatelet therapy (DAPT) alone, few data are available about the relationship between $\mathrm{FVH}$ and END and 90-day prognosis currently. In this study, we investigated the value of FVH combined with other risk factors in predicting END and short-term prognosis in AIS patients.

We present the following article in accordance with the STROBE reporting checklist (available at http://dx.doi. org/10.21037/apm-20-1175).

\section{Methods}

\section{General information}

We collected the data of AIS patients treated at the Department of Neurology of our hospital between January 2014 and December 2018. Inclusion criteria were: (I) AIS patients within 24 to 72 hours from stroke onset to magnetic resonance imaging (MRI) and contraindicated for guideline-recommended thrombolysis or thrombectomy; and (II) AIS patients treated with anticoagulation therapy, antiplatelet therapy, and oral statins. Exclusion criteria were: (I) MRI indicating conditions other than cerebral infarction, such as cerebral hemorrhage, tumors, or encephalitis; (II) bilateral infarction; (III) poor image quality cannot meet the requirements of clinical qualitative and quantitative evaluation.
We collected the general patient information, including patient age, sex, time of onset, risk factors for stroke (atrial fibrillation, systolic pressure, diastolic pressure, glucose, triglycerides, low-density lipoprotein, high-density lipoprotein), cerebral white matter hyperintensity (Fazekas grade), and significant END-related chemistry indicators (C-reactive protein, brain natriuretic peptide precursor) as reported in the literature (13-15). We also collected the NIHSS score at admission and on days 3 of admission and the 90-day modified Rankin Scale (mRS) score. END was defined when the NIHSS score increased by $\geq 2$ within 72 hours of onset (16). The patients were divided into the END group (NIHSS score increased by $\geq 2$ by day 3 of admission), and no early neurological deterioration (NEND) group (NIHSS score increased by $<2$ ). The 90-day prognosis was evaluated with the modified Rankin Scale $(\mathrm{mRS})$, and the patients were divided into the favorable prognosis group ( $\mathrm{mRS}$ score $0-2$ ) and the unfavorable prognosis group ( $\mathrm{mRS}$ score $>2$ ).

The study was conducted in accordance with the Declaration of Helsinki (as revised in 2013), and was approved by the Ethics Committee of the Second Affiliated Hospital of Nantong University (No. 2020YL025). Written informed consent was obtained from all patients.

\section{Imaging protocol}

MRI examinations were performed with 3.0-T MRI (Verio, Siemens, Germany). The MRI protocol for AIS patients included the following sequences: T1WI (TR =2,000 ms, $\mathrm{TE}=9 \mathrm{~ms}$, slice thickness $=5 \mathrm{~mm}$, interslice gap $=1 \mathrm{~mm}$, field of view $(\mathrm{FOV})=230 \times 98.8 \mathrm{~mm})$; T2WI $(\mathrm{TR}=5,500 \mathrm{~ms}$, TE $=95 \mathrm{~ms}$, slice thickness $=5 \mathrm{~mm}$, interslice gap $=1 \mathrm{~mm}, \mathrm{FOV}$ $=220 \times 100 \mathrm{~mm})$; T2-FLAIR $(\mathrm{TR}=2,000 \mathrm{~ms}$, TE $=9 \mathrm{~ms}$, slice thickness $=5 \mathrm{~mm}$, interslice gap $=1 \mathrm{~mm}$, and FOV $=230 \times 90.6 \mathrm{~mm}), \mathrm{DWI}(\mathrm{TR}=6,600 \mathrm{~ms}, \mathrm{TE}=100 \mathrm{~ms}$, slice thickness $=5.0 \mathrm{~mm}$, interslice gap $=1 \mathrm{~mm}, \mathrm{FOV}=230 \times 100 \mathrm{~mm}$, and $\mathrm{b}=1,000 \mathrm{~s} / \mathrm{mm})$, susceptibility-weighted imaging $(\mathrm{SWI})(\mathrm{TR}=28 \mathrm{~ms}, \mathrm{TE}=20 \mathrm{~ms}$, slice thickness $=1.2 \mathrm{~mm}$, no interslice gap, FOV $=230 \times 75 \mathrm{~mm}$ ), and time-of-flight magnetic resonance angiography (TOF-MRA) (TR $=21 \mathrm{~ms}$, $\mathrm{TE}=3.6 \mathrm{~ms}$, slice thickness $=0.5 \mathrm{~mm}$, interslice gap $=19.0 \mathrm{~mm}, \mathrm{FOV}=200 \times 90.6 \mathrm{~mm}$ ).

FVH was defined according to earlier studies (17), as focal, serpentine, or tube-shaped hyperintensity passing through the subarachnoid space. FVH is slow blood flow through collaterals existing within the pia mater after arterial occlusion $(10,18)$. FVH score was evaluated with 
the modified Alberta Stroke Program Early CT Score (ASPECTS). Seven cortex regions (M1 to M6, insular cortex) supplied by the middle cerebral artery were observed for FVH; one point was deducted for each region involved, with the highest score of $7(5,19)$. Multiple hypointense vessels (MHV) were evaluated with the modified ASPECTS (the highest score of 8), and one point was deducted for each region involved (20). DWI was evaluated with the conventional ASPECT score (the highest score of 10), and one point was reduced for each region involved (21). Vascular stenosis or occlusion was evaluated with TOFMRA. Additionally, the brain white matter hyperintensity was evaluated with the 6-point Fazekas scale (22). Lastly, the vascular stenosis/occlusion was defined as the occlusion or stenosis of the internal carotid artery or middle cerebral artery $\geq 50 \%$ on TOF-MRA (23) .

All images were evaluated by two experienced radiologists blindly to clinical informations. In case of discrepancy, a third, senior radiologist was consulted to reconcile the results.

\section{Statistical analysis}

SPSS v19.0 was used for statistical analysis. Continuous variables are expressed as mean \pm standard deviation and were analyzed with Student's $t$-test. Categorical variables are expressed as n (\%) and were analyzed with the MannWhitney $\mathrm{U}$ test. Ordinal variables are expressed as median [interquartile range (IQR)] and were analyzed with the chisquared $\left(\chi^{2}\right)$ test. Variables with $\mathrm{P}<0.05$ were independent variables, and END and short-term efficacy were dependent variables for logistic regression analysis. Binary stepwise regression analysis was run to analyze the odds ratio (OR) and its $95 \%$ confidence interval (CI) of primary outcomes. Non-significant variables $(\mathrm{P}>0.5)$ were successively excluded from the last model. $\mathrm{P}<0.05$ (two-sided) was considered statistically significant.

\section{Results}

A total of 267 AIS patients within 24 to 72 hours of onset who were receiving DAPT were included in this study. The average age was $66.06 \pm 11.76$ years, and the average time from onset to MRI was $44.44 \pm 16.48$ hours. The median (IQR) NIHSS score at admission was $6[5,9]$, the median DWI-ASPECTS at admission was 8 (IQR 6,9), the median FVH score was 7 (IQR 3, 7), and the median MHV-ASPECTS was 8 (IQR 6, 8). The NIHSS score at admission was higher in the END group, and the MHVASPECTS, DWI-ASPECTS, and there were lower scores in the FVH-ASPECTS (Figure 1, Table 1).

Next, we performed binary stepwise regression analysis that incorporated potential risk factors of END, including age, sex, NIHSS score at admission, DWI-ASPECTS, MHV-ASPECTS, FVH-ASPECTS, atrial fibrillation, vascular stenosis, glucose, brain natriuretic peptide precursor, and C-reactive protein. The results showed that FVH-ASPECTS (OR $=0.39,95 \%$ CI: $0.174-0.872, \mathrm{P}<0.05)$ and vascular stenosis/occlusion $(\mathrm{OR}=0.015,95 \% \mathrm{CI}$ : 0.000-0.943, $\mathrm{P}<0.05$ ) were independent risk factors of END (Table 2). The Nagelkerke $\mathrm{R}^{2}$ of the last regression model was $47.5 \%$.

Sixty-nine patients (25.84\%) experienced END, and 68 patients $(25.47 \%)$ had an adverse prognosis (90-day mRS score $>2$ ). The patients were divided into two groups (FVHpositive, FVH-negative). The $\chi^{2}$ test showed that in the FVH-positive group, the END incidence was significantly higher $(\mathrm{P}<0.001)$, with an adverse 90 -day prognosis $(\mathrm{P}<0.001)$ and a higher incidence of 90-day mRS score of 3-6 significantly (Figure 2).

\section{Discussion}

This study only included AIS patients beyond the time window for recanalization therapy who were receiving DAPT. The overall incidence of END was $25.84 \%$. It has been reported that approximately $10 \%$ of patients experience END after thrombolysis (24). In our research, we just focus on patients who are beyond the time window for recanalization therapy, and there is a lack of comparison of data on patients who underwent reperfusion therapy.

We found that the NIHSS score at admission, DWIASPECTS, FVH-ASPECTS, and MHV-ASPECTS, especially DWI-ASPECTS and FVH-ASPECTS, were correlated with END and adverse prognosis in stroke patients. A low DWI-ASPECTS was associated with a higher risk of END, which may be due to a larger infarction need more compensatory collateral circulation (25-27). The infarction volume is a known significant risk factor for END (28), and FVH may be an early imaging sign of a large infarction. FVH indicates that the brain with a decreased cerebral blood flow, and the hypoperfusion tissue may progress to an infarction without effective reperfusion (29). FVH sign may appear earlier than the hyperintensity in DWI sequences; it is a risk factor for END.

Forty-five percent of AIS patients appear FVH signs 

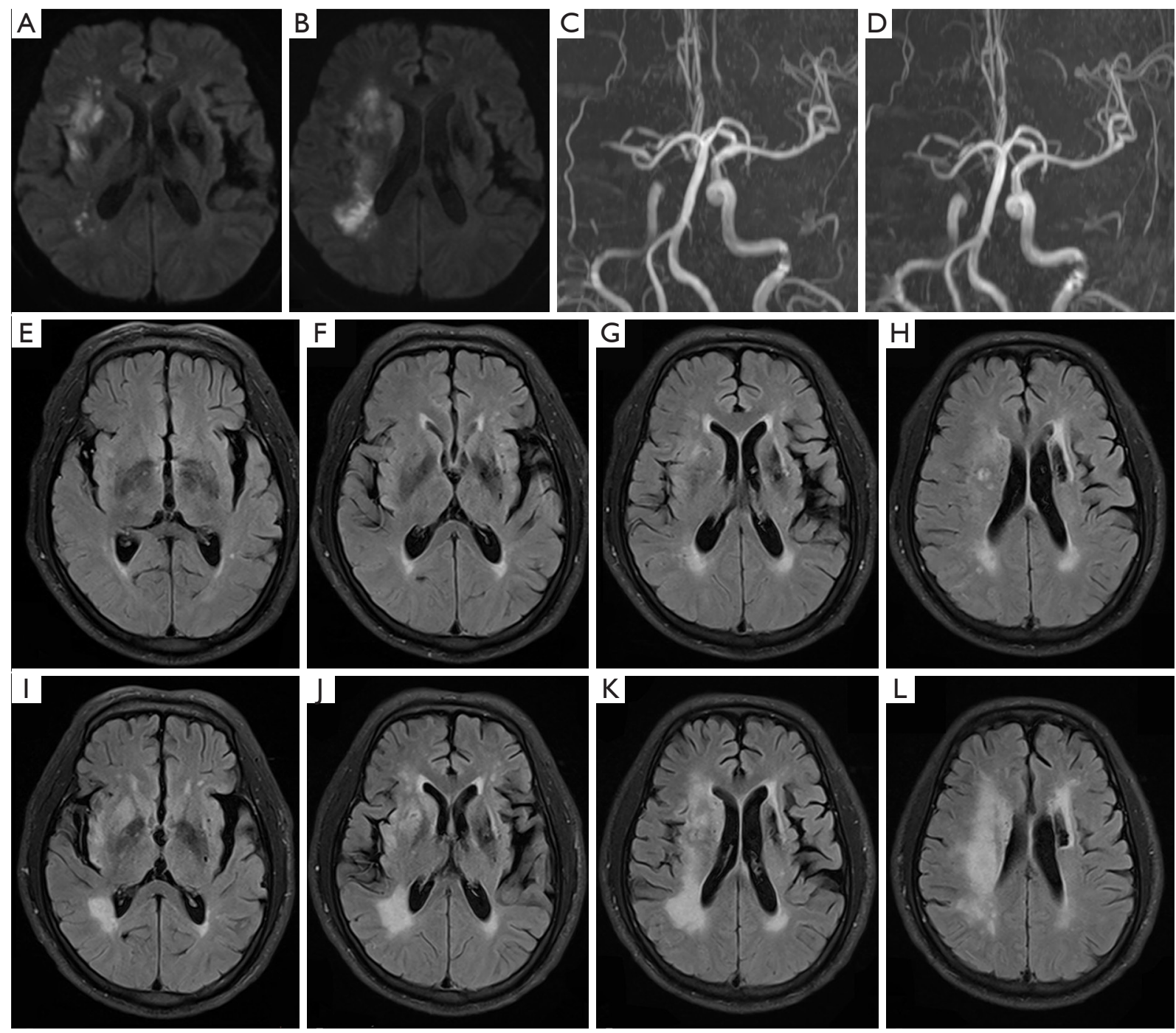

Figure 1 MRI imaging obtained 38 hours after stroke onset in a 53-year-old man, with an NIHSS score of 9 at admission and 12 on day 3 of admission, and a 90-day mRS score of 3. DWI-ASPECTS of 7 at admission MRI (A), and DWI-ASPECTS of 6 in 15 days of admission (B). A right middle cerebral artery (M1-MCA) occlusion on TOF-MRA at admission (C) and M1-MCA failed recanalization on 15 days of admission (D). FVH-ASPECTS of 3 on FLAIR imaging performed 38 hours after stroke onset (E,F,G,H), an FVH-ASPECTS of 4 at 15 days admission, the infarct progressed (I,J,K,L). mRS, modified Rankin Scale; FVH, fluid-attenuated inversion recovery (FLAIR) vascular hyperintensity.

within 24 hours of onset, and more than $90 \%$ of patients with middle cerebral artery occlusion appear FVH signs within 24 hours of onset (17) . FVH seems to be transient and disappears within 24 to 36 hours of onset in stroke patients (27). However, this study showed that 72 hours after onset, $41.94 \%$ of AIS patients still appeared FVH on MRI, which may be because the patients included in this study did not receive thrombolysis, resulting in persistent vascular occlusion or stenosis and hypoperfusion of brain tissue. Also, previous studies $(10,30)$ showed that FVH might persist for several weeks after onset, indicating persistent cerebral hemodynamic impairment in brain tissue, which indirectly confirms that FVH is a risk factor for END.

Once END occurs, it leads to severe neurological dysfunction (2). This study showed that $56.52 \%$ of END patients had an adverse 90-day prognosis, its higher than that in the NEND group. Ischemic penumbra may be 
Table 1 Baseline characteristics and radiological characteristics in patients with END and NEND

\begin{tabular}{|c|c|c|c|c|}
\hline Variables & NEND group $(n=198)$ & END group $(n=69)$ & $\mathrm{t} / \chi^{2 /} / Z$ value & $P$ value \\
\hline Sex, $M, n(\%)$ & $131(66.16)$ & $46(66.67)$ & 0.006 & 0.939 \\
\hline Onset time to $\mathrm{MRI}$, hour, mean $\pm \mathrm{SD}$ & $44.03 \pm 16.18$ & $45.63 \pm 17.37$ & 0.694 & 0.488 \\
\hline DWI-ASPECTS, median, (IQR) & $8(7,9)$ & $6(5,8)$ & -5.839 & $<0.001$ \\
\hline FVH-ASPECTS, median, (IQR) & $7(5,7)$ & $3(2,5)$ & -7.932 & $<0.001$ \\
\hline MHV-ASPECTS, median, (IQR) & $8(8,8)$ & $4(1.5,8)$ & -8.799 & $<0.001$ \\
\hline TOAST classification & & & 7.075 & 0.132 \\
\hline Large-artery atherosclerosis & 101 & 34 & & \\
\hline Other, explained & 2 & 1 & & \\
\hline Unexplained & 37 & 5 & & \\
\hline Previous infarction, $\mathrm{n}(\%)$ & $27(13.64)$ & $9(13.04)$ & 0.015 & 0.901 \\
\hline Atrial fibrillation, n (\%) & $30(15.15)$ & $18(26.09)$ & 4.150 & 0.042 \\
\hline ICA/MCA stenosis/occlusion, n (\%) & $63(31.82)$ & $54(78.26)$ & 44.831 & $<0.001$ \\
\hline WMH (Fazekas grade), median, (IQR) & $2(1,3)$ & $2(1,3)$ & -0.327 & 0.744 \\
\hline \multicolumn{5}{|l|}{ Risk factors } \\
\hline Systolic pressure, $\mathrm{mm} \mathrm{Hg}$, mean $\pm \mathrm{SD}$ & $150.11 \pm 22.77$ & $147.36 \pm 19.31$ & -0.863 & 0.389 \\
\hline High-density lipoprotein, mmol/L, mean \pm SD & $1.08 \pm 0.28$ & $1.08 \pm 0.30$ & 0.016 & 0.987 \\
\hline C-reactive protein, $\mathrm{mg} / \mathrm{L}$, mean $\pm \mathrm{SD}$ & $7.57 \pm 19.68$ & $10.34 \pm 19.23$ & 0.735 & 0.463 \\
\hline Glycated hemoglobin $(\%)$, mean \pm SD & $6.87 \pm 1.82$ & $7.49 \pm 2.00$ & 1.545 & 0.125 \\
\hline Homocysteine, $\mu$ mol/l, mean $\pm \mathrm{SD}$ & $14.10 \pm 8.95$ & $16.20 \pm 13.90$ & 1.218 & 0.225 \\
\hline Brain natriuretic peptide precursor, mean \pm SD & $837.61 \pm 1,133.08$ & $1,583.63 \pm 2,182.04$ & 1.869 & 0.066 \\
\hline 90-day mRS score (>2), n (\%) & $29(14.65)$ & $39(56.52)$ & 47.269 & $<0.001$ \\
\hline
\end{tabular}

END, early neurological deterioration; NEND, no early neurological deterioration; ASPECTS, Alberta Stroke Program Early CT Score; DWI, diffusion-weighted imaging; FVH, fluid-attenuated inversion recovery (FLAIR) vascular hyperintensity; MHV, multiple hypointense vessels; ICA, internal carotid artery; MCA, middle cerebral artery; NIHSS, National Institute of Health stroke scale; mRS, modified Rankin Scale scoring; IQR, interquartile range. 
Table 2 Last model for the binary regression with stepwise forward variable selection

\begin{tabular}{lccc}
\hline Variables & OR for END & $95 \% \mathrm{Cl}$ & $\mathrm{P}$ value \\
\hline FVH-ASPECTS & 0.390 & $0.174-0.872$ & 0.022 \\
DWI-ASPECTS & 0.588 & $0.337-1.025$ & 0.061 \\
Vascular stenosis/occlusion & 0.015 & $0.000-0.943$ & 0.047 \\
\hline
\end{tabular}

END, early neurological deterioration; OR, odds ratio; $95 \% \mathrm{Cl}, 95 \%$ confidence interval.

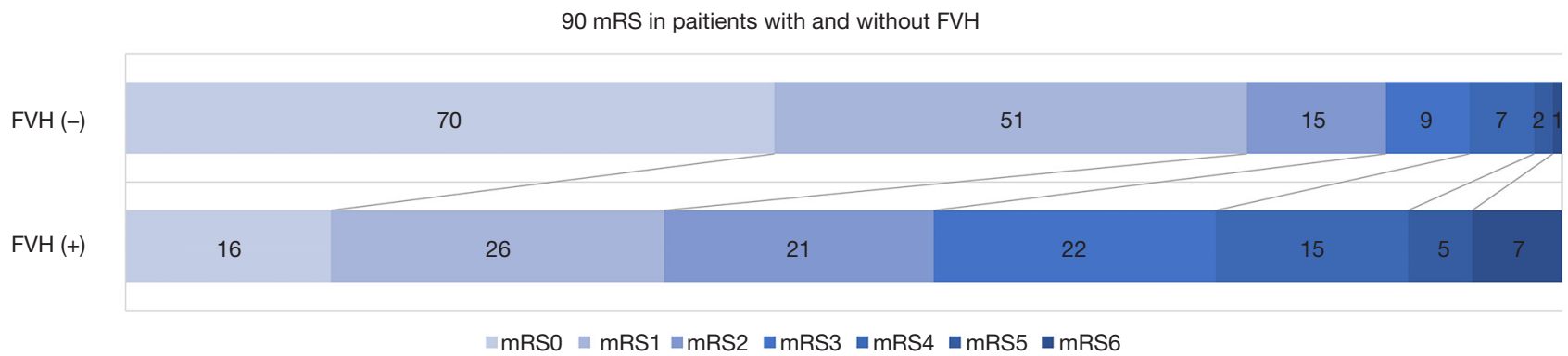

Figure 2 Functional outcomes at 90 days, according to the mRS scores in FVH-positive (FVH+) and FVH-negative (FVH-) group. mRS, modified Rankin Scale; FVH, fluid-attenuated inversion recovery (FLAIR) vascular hyperintensity.

present up to 36 hours and may progress to an infarction without effective reperfusion (31), which indirectly suggests that 24 hours may still be the early stage of stroke progression. Hakimelahi et al. (32) showed no significant correlation between the infarct volume and the onset of stroke. Other factors may affect the scope of infarction, and the variation in cerebral perfusion because of the state of collateral circulation may be the ultimate cause of varying severity of stroke. A case report showed that 37 hours after onset, patient with occlusion of the anterior circulation still had favorable prognosis after effective endovascular treatment (33). END may reflect the underlying pathophysiological process, but it is possible to be prevented. Therefore, it is essential to evaluate the risk of END early and administer individualized therapy in stroke patients. Further research is needed in patients beyond the time window for recanalization therapy.

In this study, univariate analysis showed that MHVASPECTS was correlated with END, and a lower MHVASPECTS was associated with a higher risk of END. MHV reflects hypoperfusion of brain tissues, and patients with MHV are prone to the neurological deterioration (34). However, our binary stepwise regression analysis showed that MHV-ASPECTS was not an independent risk factor for END. We surmise that MHV may be related to deoxyhemoglobin concentration. Furthermore, it may also be related to the ratio of deoxyhemoglobin to oxyhemoglobin, indicating the oxygen demand of ischemic brain tissue, rather than the state of collateral circulation (20). Further research is needed to investigate the relationship between MHV and END.

This study has some limitations; it is a retrospective, single-center study with potential recall bias. The patients without $\mathrm{FVH}$ are more than patients with $\mathrm{FVH}$ significantly, which may led to a certain bias. We only performed semi-quantitative analysis on FVH. We may be able to perform further quantitative analysis with upgraded software. No guidelines have been developed to support reperfusion in patients beyond the time window for recanalization therapy. For stroke patients beyond the time window for recanalization therapy, who may develop END, it is vital to conduct a comprehensive clinical evaluation and administer early clinical interventions with individualized therapy to improve patient outcomes. More research is needed to investigate this topic.

\section{Conclusions}

For AIS patients beyond the time window for recanalization therapy who are receiving DAPT, a lower FVH-ASPECTS is associated with a higher risk of END. For patients with vascular occlusion/stenosis, FVH may predict END and 
unfavorable prognosis.

\section{Acknowledgments}

Funding: This study was funded by the grant of Science Foundation of Nantong (MS12018087, MS12018042, HS2019002) and the grant of Science Foundation of Jiangsu Commission of Health (H2019057)

\section{Footnote}

Reporting Checklist: The authors have completed the STROBE reporting checklist. Available at http://dx.doi. org/10.21037/apm-20-1175

Data Sharing Statement: Available at http://dx.doi. org/10.21037/apm-20-1175

Conflicts of Interest: All authors have completed the ICMJE uniform disclosure form (available at http://dx.doi. org/10.21037/apm-20-1175). The authors have no conflicts of interest to declare.

Ethical Statement: The authors are accountable for all aspects of the work in ensuring that questions related to the accuracy or integrity of any part of the work are appropriately investigated and resolved. The study was conducted in accordance with the Declaration of Helsinki (as revised in 2013), and was approved by the Ethics Committee of the Second Affiliated Hospital of Nantong University (No. 2020YL025). Written informed consent was obtained from all patients.

Open Access Statement: This is an Open Access article distributed in accordance with the Creative Commons Attribution-NonCommercial-NoDerivs 4.0 International License (CC BY-NC-ND 4.0), which permits the noncommercial replication and distribution of the article with the strict proviso that no changes or edits are made and the original work is properly cited (including links to both the formal publication through the relevant DOI and the license). See: https://creativecommons.org/licenses/by-nc-nd/4.0/.

\section{References}

1. Sedlaczek O, Caplan L, Hennerici M. Impaired washout-embolism and ischemic stroke: further examples and proof of concept. Cerebrovasc Dis 2005;19:396-401.
2. Seners P, Turc G, Oppenheim C, et al. Incidence, causes and predictors of neurological deterioration occurring within $24 \mathrm{~h}$ following acute ischaemic stroke: a systematic review with pathophysiological implications. J Neurol Neurosurg Psychiatry 2015;86:87-94.

3. Seners P, Baron JC. Revisiting 'progressive stroke': incidence, predictors, pathophysiology, and management of unexplained early neurological deterioration following acute ischemic stroke. J Neurol 2018;265:216-25.

4. Alawneh JA, Moustafa RR, Baron JC. Hemodynamic factors and perfusion abnormalities in early neurological deterioration. Stroke 2009;40:e443-50.

5. Nave AH, Kufner A, Bücke P, et al. Hyperintense Vessels, Collateralization, and Functional Outcome in Patients With Stroke Receiving Endovascular Treatment. Stroke 2018;49:675-81.

6. Legrand L, Tisserand M, Turc G, et al. Fluid-Attenuated Inversion Recovery Vascular Hyperintensities-DiffusionWeighted Imaging Mismatch Identifies Acute Stroke Patients Most Likely to Benefit From Recanalization. Stroke 2016;47:424-7.

7. Gu HQ, Yang X, Rao ZZ, et al. Disparities in outcomes associated with rural-urban insurance status in China among inpatient women with stroke: a registry-based cohort study. Ann Transl Med 2019;7:426.

8. Ebinger M, Kufner A, Galinovic I, et al. Fluid-attenuated inversion recovery images and stroke outcome after thrombolysis. Stroke 2012;43:539-42.

9. Legrand L, Turc G, Edjlali M, et al. Benefit from revascularization after thrombectomy according to FLAIR vascular hyperintensities-DWI mismatch. Eur Radiol 2019;29:5567-76.

10. Sanossian N, Saver JL, Alger JR, et al. Angiography reveals that fluid-attenuated inversion recovery vascular hyperintensities are due to slow flow, not thrombus. AJNR Am J Neuroradiol 2009;30:564-8.

11. Haussen DC, Koch S, Saraf-Lavi E, et al. FLAIR distal hyperintense vessels as a marker of perfusiondiffusion mismatch in acute stroke. J Neuroimaging 2013;23:397-400.

12. Bouchez L, Sztajzel R, Vargas MI, et al. CT imaging selection in acute stroke. Eur J Radiol 2017;96:153-61.

13. Leigh R, Zaidat OO, Suri MF, et al. Predictors of hyperacute clinical worsening in ischemic stroke patients receiving thrombolytic therapy. Stroke 2004;35:1903-7.

14. Seo WK, Seok HY, Kim JH, et al. C-reactive protein is a predictor of early neurologic deterioration in acute ischemic stroke. J Stroke Cerebrovasc Dis 2012;21:181-6. 
15. Huang GX, Ji XM, Ding YC, et al. Association between serum cystatin $\mathrm{C}$ levels and the severity or potential risk factors of acute ischemic stroke. Neurol Res 2016;38:518-23.

16. Jeong HG, Kim BJ, Yang MH, et al. Neuroimaging markers for early neurologic deterioration in single small subcortical infarction. Stroke 2015;46:687-91.

17. Azizyan A, Sanossian N, Mogensen MA, et al. Fluidattenuated inversion recovery vascular hyperintensities: an important imaging marker for cerebrovascular disease. AJNR Am J Neuroradiol 2011;32:1771-5.

18. Kufner A, Galinovic I, Ambrosi V, et al. Hyperintense Vessels on FLAIR: Hemodynamic Correlates and Response to Thrombolysis. AJNR Am J Neuroradiol 2015;36:426-30.

19. Ahn SJ, Suh SH, Lee KY, et al. Hyperintense Vessels on T2-PROPELLER-FLAIR in Patients with Acute MCA Stroke: Prediction of Arterial Stenosis and Perfusion Abnormality. AJNR Am J Neuroradiol 2015;36:2042-7.

20. Park MG, Yang TI, Oh SJ, et al. Multiple hypointense vessels on susceptibility-weighted imaging in acute ischemic stroke: surrogate marker of oxygen extraction fraction in penumbra. Cerebrovasc Dis 2014;38:254-61.

21. Barber PA, Demchuk AM, Zhang J, et al. Validity and reliability of a quantitative computed tomography score in predicting outcome of hyperacute stroke before thrombolytic therapy. ASPECTS Study Group. Alberta Stroke Programme Early CT Score. Lancet 2000;355:1670-4.

22. Helenius J, Goddeau RP Jr, Moonis M, et al. Impact of Leukoaraiosis Burden on Hemispheric Lateralization of the National Institutes of Health Stroke Scale Deficit in Acute Ischemic Stroke. Stroke 2016;47:24-30.

23. Chimowitz MI, Lynn MJ, Howlett-Smith H, et al. Comparison of warfarin and aspirin for symptomatic intracranial arterial stenosis. N Engl J Med 2005;352:1305-16.

24. Seners P, Turc G, Tisserand M, et al. Unexplained early neurological deterioration after intravenous thrombolysis: incidence, predictors, and associated factors. Stroke 2014;45:2004-9.

25. Cheng B, Ebinger M, Kufner A, et al. Hyperintense vessels on acute stroke fluid-attenuated inversion recovery imaging: associations with clinical and other MRI findings. Stroke 2012;43:2957-61.

26. Gawlitza M, Gragert J, Quäschling U, et al. FLAIRhyperintense vessel sign, diffusion-perfusion mismatch and infarct growth in acute ischemic stroke without vascular recanalisation therapy. J Neuroradiol 2014;41:227-33.

27. Hohenhaus M, Schmidt WU, Brunecker P, et al. FLAIR vascular hyperintensities in acute ICA and MCA infarction: a marker for mismatch and stroke severity. Cerebrovasc Dis 2012;34:63-9.

28. Arenillas JF, Rovira A, Molina CA, et al. Prediction of early neurological deterioration using diffusion- and perfusion-weighted imaging in hyperacute middle cerebral artery ischemic stroke. Stroke 2002;33:2197-203.

29. Nam KW, Kwon HM, Park SW, et al. Distal hyperintense vessel sign is associated with neurological deterioration in acute ischaemic stroke. Eur J Neurol 2017;24:617-23.

30. Essig M, von Kummer R, Egelhof T, et al. Vascular MR contrast enhancement in cerebrovascular disease. AJNR Am J Neuroradiol 1996;17:887-94.

31. Ni L, Li J, Li W, et al. The value of resting-state functional MRI in subacute ischemic stroke: comparison with dynamic susceptibility contrast-enhanced perfusion MRI. Sci Rep 2017;7:41586.

32. Hakimelahi R, Vachha BA, Copen WA, et al. Time and diffusion lesion size in major anterior circulation ischemic strokes. Stroke 2014;45:2936-41.

33. So C, Chaudhry N, Gandhi D, et al. Endovascular Thrombectomy in Acute-Onset Ischemic Stroke - beyond the Standard Time Windows: A Case Report and a Review of the Literature. Case Rep Neurol 2018;10:279-85.

34. Luo Y, Gong Z, Zhou Y, et al. Increased susceptibility of asymmetrically prominent cortical veins correlates with misery perfusion in patients with occlusion of the middle cerebral artery. Eur Radiol 2017;27:2381-90.
Cite this article as: Zhu L, Gong S, Zhu X, Zhang R, Ren $\mathrm{K}$, Zhu Z, Wang T, Xing W. FLAIR vascular hyperintensity: an unfavorable marker of early neurological deterioration and short-term prognosis in acute ischemic stroke patients. Ann Palliat Med 2020;9(5):3144-3151. doi: 10.21037/apm-20-1175 